\title{
On the Hermite-Hadamard Inequality and Other Integral Inequalities Involving Several Functions
}

\author{
Banyat Sroysang \\ Department of Mathematics and Statistics, Faculty of Science and Technology, Thammasat University, Pathumthani 12121, Thailand \\ Correspondence should be addressed to Banyat Sroysang; banyat@mathstat.sci.tu.ac.th
}

Received 18 March 2013; Accepted 14 May 2013

Academic Editor: Nelson Merentes

Copyright (c) 2013 Banyat Sroysang. This is an open access article distributed under the Creative Commons Attribution License, which permits unrestricted use, distribution, and reproduction in any medium, provided the original work is properly cited.

We present some new Hermite-Hadamard-type inequalities and other integral inequalities involving several functions.

\section{Introduction and Preliminaries}

In 2003, Pachpatte [1] gave some Hermite-Hadamard-type inequalities involving two convex functions, and then Pachpatte [2] also gave, in 2004, some Hermite-Hadamard-type inequalities involving two log-convex functions. In 2007, Kirmaci et al. [3] gave some Hadamard-type inequalities involving $s$-convex functions. In 2008, Bakula et al. [4] presented some Hadamard-type inequalities involving $\mathrm{m}$ convex functions and $(\alpha, m)$-convex functions. In 2010, Set et al. [5] gave some new Hermite-Hadamard-type inequalities and other integral inequalities involving two functions. More details about results proved in [5] will be given in Section 2. In this paper, we present more general Hermite-Hadamard-type inequalities and some integral inequalities involving several functions.

Let $f:[a, b] \rightarrow \mathbb{R}$ and $p \geq 1$. The $p$-norm of the function $f$ on $[a, b]$ is defined by

$$
\|f\|_{p}= \begin{cases}\left(\int_{a}^{b}|f(x)|^{p} d x\right)^{1 / p} ; & 1 \leq p<\infty \\ \sup _{x \in[a, b]}|f(x)| ; & p=+\infty .\end{cases}
$$

Below we recall few well-known inequalities that will be useful in the proofs of our results.
Hermite-Hadamard's Inequality (see [6-8]). If $f$ is a convex function on $[a, b]$, then

$$
f\left(\frac{a+b}{2}\right) \leq \frac{1}{b-a} \int_{a}^{b} f(x) d x \leq \frac{f(a)+f(b)}{2} .
$$

If $f$ is a concave function on $[a, b]$, then

$$
\frac{f(a)+f(b)}{2} \leq \frac{1}{b-a} \int_{a}^{b} f(x) d x \leq f\left(\frac{a+b}{2}\right) .
$$

Barnes-Gugunova-Levin Inequality (see [9-11]). If $f$ and $g$ are nonnegative concave functions on $[a, b]$, and if $p, q>1$, then

$$
\begin{gathered}
\left(\int_{a}^{b} f^{p}(x) d x\right)^{1 / p}\left(\int_{a}^{b} g^{q}(x) d x\right)^{1 / q} \\
\leq B(p, q) \int_{a}^{b} f(x) g(x) d x
\end{gathered}
$$

where

$$
B(p, q)=\frac{6(b-a)^{1 / p+1 / q-1}}{(p+1)^{1 / p}(q+1)^{1 / q}} .
$$


The Power-Mean Inequality (see [12]). Let $x_{1}, x_{2}, \ldots, x_{n}, p_{1}$, $p_{2}, \ldots, p_{n}>0$ and let $r \in \mathbb{R} \cup\{-\infty,+\infty\}$. Then

$$
M_{n}^{[r]}= \begin{cases}\left(\frac{\sum_{i=1}^{n} p_{i} x_{i}^{r}}{\sum_{i=1}^{n} p_{i}}\right)^{1 / r} ; & r \neq 0, \pm \infty, \\ \left(\prod_{i=1}^{n} x_{i}^{1} p_{i}\right)^{1 / \sum_{i=1}^{n} p_{i}} ; & r=0, \\ \max \left\{x_{1}, x_{2}, \ldots, x_{n}\right\} ; & r=\infty, \\ \min \left\{x_{1}, x_{2}, \ldots, x_{n}\right\} ; & r=-\infty .\end{cases}
$$

Notice that if $-\infty \leq r<s \leq+\infty$ then

$$
M_{n}^{[r]} \leq M_{n}^{[s]}
$$

A Generalization of Hölder Integral Inequality. For any $p_{1}$, $p_{2}, \ldots, p_{n}>1, \sum_{i=1}^{n}\left(1 / p_{i}\right)=1$, if $f_{1}, f_{2}, \ldots, f_{n}$ are nonnegative functions on $[a, b]$ and if $f_{1}^{p_{1}}, f_{2}^{p_{2}}, \ldots, f_{n}^{p_{n}}$ are integrable functions on $[a, b]$, then

$$
\int_{a}^{b}\left(\prod_{i=1}^{n} f_{i}(x)\right) d x \leq \prod_{i=1}^{n}\left(\int_{a}^{b} f_{i}^{p_{i}}(x) d x\right)^{1 / p_{i}} .
$$

A Generalization of Minkowski Integral Inequality. If $p \geq 1$ and if $f_{1}, f_{2}, \ldots, f_{n}$ are non-negative functions on $[a, b]$ such that $0<\int_{a}^{b} f_{i}^{p}(x) d x<\infty$ for all $i=1,2, \ldots, n$, then

$$
\left(\int_{a}^{b}\left(\sum_{i=1}^{n} f_{i}(x)\right)^{p} d x\right)^{1 / p} \leq \sum_{i=1}^{n}\left(\int_{a}^{b} f_{i}^{p}(x) d x\right)^{1 / p}
$$

A Generalization of Young Inequality. If $x_{1}, x_{2}, \ldots, x_{n} \geq 0$ and $p_{1}, p_{2}, \ldots, p_{n}>1, \sum_{i=1}^{n}\left(1 / p_{i}\right)=1$, then

$$
\prod_{i=1}^{n} x_{i} \leq \sum_{i=1}^{n} \frac{x^{p_{i}}}{p_{i}}
$$

To prove results, we refer to the following lemma.

Lemma 1 (see [13]). If $p \geq 1$ and if $f$ and $g$ are positive functions on $[a, b]$ such that $0<m \leq f(x) / g(x) \leq M$ for all $x \in[a, b]$, then

$$
\begin{aligned}
& \left(\int_{a}^{b} f^{p}(x) d x\right)^{1 / p} \leq \frac{M}{M+1}\left(\int_{a}^{b}(f(x)+g(x))^{p} d x\right)^{1 / p}, \\
& \left(\int_{a}^{b} g^{p}(x) d x\right)^{1 / p} \leq \frac{1}{m+1}\left(\int_{a}^{b}(f(x)+g(x))^{p} d x\right)^{1 / p} .
\end{aligned}
$$

Proof. Let $p \geq 1$. Assume that $f$ and $g$ are positive functions on $[a, b]$ such that $0<m \leq f(x) / g(x) \leq M$ for all $x \in[a, b]$. Then

$$
\begin{aligned}
f & \leq M g=M(f+g)-M f \\
g & \leq \frac{1}{m} f=\frac{1}{m}(f+g)-\frac{1}{m} g .
\end{aligned}
$$

Then

$$
\begin{gathered}
(M+1)^{p} f^{p} \leq M^{p}(f+g)^{p}, \\
\left(\frac{1}{m}+1\right)^{p} g^{p} \leq\left(\frac{1}{m}\right)^{p}(f+g)^{p} .
\end{gathered}
$$

Thus,

$$
\begin{aligned}
& \left(\int_{a}^{b} f^{p}(x) d x\right)^{1 / p} \leq \frac{M}{M+1}\left(\int_{a}^{b}(f(x)+g(x))^{p} d x\right)^{1 / p}, \\
& \left(\int_{a}^{b} g^{p}(x) d x\right)^{1 / p} \leq \frac{1}{m+1}\left(\int_{a}^{b}(f(x)+g(x))^{p} d x\right)^{1 / p} .
\end{aligned}
$$

\section{Main Results}

We start this section with the following.

Theorem 2. Let $n$ be a positive even integer and $p_{1}, p_{2}, \ldots$, $p_{n}>1$ and let $f_{1}, f_{2}, \ldots, f_{n}$ be non-negative functions on $[a, b]$ such that $f_{1}^{p_{1}}, f_{2}^{p_{2}}, \ldots, f_{n}^{p_{n}}$ are concave on $[a, b]$. Then

$\prod_{i=1}^{n}\left(\frac{f_{i}(a)+f_{i}(b)}{2}\right)$

$\leq \frac{1}{(b-a)^{\sum_{i=1}^{n}\left(1 / p_{i}\right)}}\left(\prod_{i=1}^{n / 2} B\left(p_{2 i-1}, p_{2 i}\right) \int_{a}^{b} f_{2 i-1}(x) f_{2 i}(x) d x\right)$,

where

$$
B\left(p_{2 i-1}, p_{2 i}\right)=\frac{6(b-a)^{1 / p_{2 i-1}+1 / p_{2 i}-1}}{\left(p_{2 i-1}+1\right)^{1 / p_{2 i-1}}\left(p_{2 i}+1\right)^{1 / p_{2 i}}}
$$

for all $i=1, \ldots, n / 2$. Moreover, if $\sum_{i=1}^{n}\left(1 / p_{i}\right)=1$, then

$$
\frac{1}{b-a} \int_{a}^{b}\left(\prod_{i=1}^{n} f_{i}(x)\right) d x \leq \prod_{i=1}^{n} f_{i}\left(\frac{a+b}{2}\right) \text {. }
$$

Proof. Applying the inequality (3) with $f_{i}^{p_{i}}$, for any $i=$ $1, \ldots, n$, we get

$$
\frac{f_{i}^{p_{i}}(a)+f_{i}^{p_{i}}(b)}{2} \leq \frac{1}{b-a} \int_{a}^{b} f_{i}^{p_{i}}(x) d x \leq f_{i}^{p_{i}}\left(\frac{a+b}{2}\right),
$$

and, consequently,

$$
\begin{aligned}
& \left(\frac{f_{i}^{p_{i}}(a)+f_{i}^{p_{i}}(b)}{2}\right)^{1 / p_{i}} \\
& \quad \leq \frac{1}{(b-a)^{1 / p_{i}}}\left(\int_{a}^{b} f_{i}^{p_{i}}(x) d x\right)^{1 / p_{i}} \leq f_{i}\left(\frac{a+b}{2}\right)
\end{aligned}
$$


By the Barnes-Gudunova-Levin inequality (4), it follows that

$$
\begin{aligned}
& \prod_{i=1}^{n}\left(\frac{f_{i}^{p_{i}}(a)+f_{i}^{p_{i}}(b)}{2}\right)^{1 / p_{i}} \\
& \leq \frac{1}{(b-a)^{\sum_{i=1}^{n}\left(1 / p_{i}\right)}} \prod_{i=1}^{n}\left(\int_{a}^{b} f_{i}^{p_{i}}(x) d x\right)^{1 / p_{i}} \\
& =\frac{1}{(b-a)^{\sum_{i=1}^{n}\left(1 / p_{i}\right)}} \prod_{i=1}^{n / 2}\left(\int_{a}^{b} f_{2 i-1}^{p_{2 i-1}}(x) d x\right)^{1 / p_{2 i-1}} \\
& \quad \times\left(\int_{a}^{b} f_{2 i}^{p_{2 i}}(x) d x\right)^{1 / p_{2 i}} \\
& \leq \frac{1}{(b-a)^{\sum_{i=1}^{n}\left(1 / p_{i}\right)}}\left(\prod_{i=1}^{n / 2} B\left(p_{2 i-1}, p_{2 i}\right) \int_{a}^{b} f_{2 i-1}(x) f_{2 i}(x) d x\right),
\end{aligned}
$$

where

$$
B\left(p_{2 i-1}, p_{2 i}\right)=\frac{6(b-a)^{1 / p_{2 i-1}+1 / p_{2 i}-1}}{\left(p_{2 i-1}+1\right)^{1 / p_{2 i-1}}\left(p_{2 i}+1\right)^{1 / p_{2 i}}}
$$

for all $i=1, \ldots, n / 2$.

By the power-mean inequality (7), we have

$$
\left(\frac{f_{i}^{p_{i}}(a)+f_{i}^{p_{i}}(b)}{2}\right)^{1 / p_{i}} \geq \frac{f_{i}(a)+f_{i}(b)}{2}
$$

for all $i=1,2, \ldots, n$.

This implies the inequality (15).

Next, we assume that $\sum_{i=1}^{n}\left(1 / p_{i}\right)=1$. By the inequality (19) and the generalized Hölder inequality, we obtain that

$$
\begin{aligned}
\prod_{i=1}^{n} f_{i}\left(\frac{a+b}{2}\right) & \geq \frac{1}{(b-a)^{\sum_{i=1}^{n}\left(1 / p_{i}\right)}} \prod_{i=1}^{n}\left(\int_{a}^{b} f_{i}^{p_{i}}(x) d x\right)^{1 / p_{i}} \\
& =\frac{1}{b-a} \prod_{i=1}^{n}\left(\int_{a}^{b} f_{i}^{p_{i}}(x) d x\right)^{1 / p_{i}} \\
& \geq \frac{1}{b-a} \int_{a}^{b}\left(\prod_{i=1}^{n} f_{i}(x)\right) d x .
\end{aligned}
$$

This proof is completed.

It is easy to notice that if we put $n=2$ in Theorem 2 then we get the following.

Corollary 3 (see [5]). Let $p, q>1$ and let $f, g$ be non-negative functions on $[a, b]$ such that $f^{p}, g^{q}$ are concave on $[a, b]$. Then

$$
\begin{aligned}
& \left(\frac{f(a)+f(b)}{2}\right)\left(\frac{g(a)+g(b)}{2}\right) \\
& \quad \leq \frac{1}{(b-a)^{1 / p+1 / q}}\left(B(p, q) \int_{a}^{b} f(x) g(x) d x\right),
\end{aligned}
$$

where

$$
B(p, q)=\frac{6(b-a)^{1 / p+1 / q-1}}{(p+1)^{1 / p}(q+1)^{1 / q}} .
$$

Moreover, if $1 / p+1 / q=1$, then

$$
\frac{1}{b-a} \int_{a}^{b} f(x) g(x) d x \leq f\left(\frac{a+b}{2}\right) g\left(\frac{a+b}{2}\right) .
$$

Theorem 4. Let $p \geq 1$ and $n$ be a positive integer such that $n \geq 2$ and let $f_{1}, f_{2}, \ldots, f_{n}$ be positive functions on $[a, b]$ such that the functions $f_{1}^{p}, f_{2}^{p}, \ldots, f_{n}^{p}$ are integrable functions on $[a, b], 0<\int_{a}^{b} f_{i}^{p}(x) d x<\infty$ for all $i=1,2, \ldots, n$, and

$$
0<m_{i} \leq \frac{f_{i}(x)}{f_{i+1}(x)} \leq M_{i}
$$

for all $x \in[a, b]$ and for all $i=1, \ldots, n-1$. Then

$$
\frac{\left(\sum_{i=1}^{n}\left\|f_{i}\right\|_{p}\right)^{n}}{\prod_{i=1}^{n}\left\|f_{i}\right\|_{p}} \geq \frac{1}{\prod_{i=1}^{n} s_{i}},
$$

where

$$
\begin{gathered}
s_{1}=\frac{M_{1}}{M_{1}+1}, \quad s_{n}=\frac{1}{m_{n-1}+1}, \\
s_{i}=\min \left\{\frac{1}{m_{i-1}+1}, \frac{M_{i}}{M_{i}+1}\right\}
\end{gathered}
$$

for all $1<i<n$.

Proof. By Lemma 1, we have

$$
\begin{aligned}
& \left(\int_{a}^{b} f_{i}^{p}(x) d x\right)^{1 / p} \leq \frac{M_{i}}{M_{i}+1}\left(\int_{a}^{b}\left(f_{i}(x)+f_{i+1}(x)\right)^{p} d x\right)^{1 / p}, \\
& \left(\int_{a}^{b} f_{i+1}^{p}(x) d x\right)^{1 / p} \leq \frac{1}{m_{i}+1}\left(\int_{a}^{b}\left(f_{i}(x)+f_{i+1}(x)\right)^{p} d x\right)^{1 / p}
\end{aligned}
$$

for all $i=1, \ldots, n-1$.

Then

$$
\begin{aligned}
& \left(\int_{a}^{b} f_{i}^{p}(x) d x\right)^{1 / p} \leq \frac{M_{i}}{M_{i}+1}\left(\int_{a}^{b}\left(\sum_{j=1}^{n} f_{j}(x)\right)^{p} d x\right)^{1 / p}, \\
& \left(\int_{a}^{b} f_{i+1}^{p}(x) d x\right)^{1 / p} \leq \frac{1}{m_{i}+1}\left(\int_{a}^{b}\left(\sum_{j=1}^{n} f_{j}(x)\right)^{p} d x\right)^{1 / p}
\end{aligned}
$$

for all $i=1, \ldots, n-1$.

Let $s_{1}=M_{1} /\left(M_{1}+1\right), s_{n}=1 /\left(m_{n-1}+1\right)$, and $s_{i}=$ $\min \left\{1 /\left(m_{i-1}+1\right), M_{i} /\left(M_{i}+1\right)\right\}$ for all $1<i<n$. 
It follows that

$$
\begin{aligned}
& \left(\int_{a}^{b} f_{1}^{p}(x) d x\right)^{1 / p} \leq s_{1}\left(\int_{a}^{b}\left(\sum_{j=1}^{n} f_{j}(x)\right)^{p} d x\right)^{1 / p}, \\
& \left(\int_{a}^{b} f_{n}^{p}(x) d x\right)^{1 / p} \leq s_{n}\left(\int_{a}^{b}\left(\sum_{j=1}^{n} f_{j}(x)\right)^{p} d x\right)^{1 / p}, \\
& \left(\int_{a}^{b} f_{i}^{p}(x) d x\right)^{1 / p} \leq s_{i}\left(\int_{a}^{b}\left(\sum_{j=1}^{n} f_{j}(x)\right)^{p} d x\right)^{1 / p}
\end{aligned}
$$

for all $1<i<n$.

By multiplying the above inequalities and the generalized Minkowski inequality, we obtain that

$$
\begin{aligned}
& \prod_{i=1}^{n}\left(\int_{a}^{b} f_{i}^{p}(x) d x\right)^{1 / p} \\
& \quad \leq\left(\prod_{i=1}^{n} s_{i}\right)\left(\left(\int_{a}^{b}\left(\sum_{j=1}^{n} f_{j}(x)\right)^{p} d x\right)^{1 / p}\right)^{n} \\
& \quad \leq\left(\prod_{i=1}^{n} s_{i}\right)\left(\sum_{j=1}^{n}\left(\int_{a}^{b} f_{j}^{p}(x) d x\right)^{1 / p}\right)^{n} .
\end{aligned}
$$

Then

$$
\prod_{i=1}^{n}\left\|f_{i}\right\|_{p} \leq\left(\prod_{i=1}^{n} s_{i}\right)\left(\sum_{j=1}^{n}\left\|f_{i}\right\|_{p}\right)^{n} .
$$

This implies the inequality (28).

Notice that from above theorem one can easily get the following.

Corollary 5 (see [5]). Let $p \geq 1$ and let $f, g$ be positive functions on $[a, b]$ such that $0<\int_{a}^{b} f^{p}(x) d x<\infty, 0<$ $\int_{a}^{b} g^{p}(x) d x<\infty$, and

$$
0<m \leq \frac{f(x)}{g(x)} \leq M
$$

for all $x \in[a, b]$. Then

$$
\frac{\|f\|_{p}^{2}+\|g\|_{p}^{2}}{\|f\|_{p}\|g\|_{p}} \geq \frac{1}{s}-2
$$

where $s=M /(M+1)(m+1)$.

Proof. By Theorem 4 where $n=2$, we have

$$
\frac{\left(\|f\|_{p}+\|g\|_{p}\right)^{2}}{\|f\|_{p}\|g\|_{p}} \geq \frac{1}{s_{1} s_{2}}
$$

where

$$
s_{1}=\frac{M}{M+1}, \quad s_{2}=\frac{1}{m+1} .
$$

Let $s=s_{1} s_{2}$. Then

$$
\begin{aligned}
\frac{1}{s} & \leq \frac{\left(\|f\|_{p}+\|g\|_{p}\right)^{2}}{\|f\|_{p}\|g\|_{p}} \\
& =\frac{\|f\|_{p}^{2}+2\|f\|_{p}\|g\|_{p}+\|g\|_{p}^{2}}{\|f\|_{p}\|g\|_{p}} \\
& =\frac{\|f\|_{p}^{2}+\|g\|_{p}^{2}}{\|f\|_{p}\|g\|_{p}}+2 .
\end{aligned}
$$

This implies the inequality (36).

Theorem 6. Let $n$ be a positive integer such that $n \geq 2$ and $p_{1}, p_{2}, \ldots, p_{n}>1$ and let $f_{1}, f_{2}, \ldots, f_{n}$ be non-negative functions on $[a, b]$ such that $f_{1}^{p_{1}}, f_{2}^{p_{2}}, \ldots, f_{n}^{p_{n}}$ are concave on $[a, b]$. Then

$$
\prod_{i=1}^{n}\left(f_{i}(a)+f_{i}(b)\right)^{p_{i}} \leq \frac{2^{\sum_{i=1}^{n} p_{i}}}{(b-a)^{n}} \prod_{i=1}^{n}\left\|f_{i}\right\|_{p_{i}}^{p_{i}}
$$

Proof. Using the inequality (3) with $f_{i}^{p_{i}}$, for any $i=1, \ldots, n$, we obtain

$$
\frac{f_{i}^{p_{i}}(a)+f_{i}^{p_{i}}(b)}{2} \leq \frac{1}{b-a} \int_{a}^{b} f_{i}^{p_{i}}(x) d x .
$$

Then

$$
\prod_{i=1}^{n}\left(\frac{f_{i}^{p_{i}}(a)+f_{i}^{p_{i}}(b)}{2}\right) \leq \frac{1}{(b-a)^{n}} \prod_{i=1}^{n}\left(\int_{a}^{b} f_{i}^{p_{i}}(x) d x\right) .
$$

By the power-mean inequality (7), we have

$$
\left(\frac{f_{i}^{p_{i}}(a)+f_{i}^{p_{i}}(b)}{2}\right)^{1 / p_{i}} \geq \frac{f_{i}(a)+f_{i}(b)}{2}
$$

so

$$
\frac{f_{i}^{p_{i}}(a)+f_{i}^{p_{i}}(b)}{2} \geq \frac{\left(f_{i}(a)+f_{i}(b)\right)^{p_{i}}}{2^{p_{i}}}
$$

for all $i=1,2, \ldots, n$.

Then

$$
\begin{aligned}
\frac{1}{(b-a)^{n}} \prod_{i=1}^{n}\left(\int_{a}^{b} f_{i}^{p_{i}}(x) d x\right) & \geq \prod_{i=1}^{n}\left(\frac{\left(f_{i}(a)+f_{i}(b)\right)^{p_{i}}}{2^{p_{i}}}\right) \\
& =\frac{\prod_{i=1}^{n}\left(f_{i}(a)+f_{i}(b)\right)^{p_{i}}}{2^{\sum_{i=1}^{n} p_{i}}} .
\end{aligned}
$$

This implies the inequality (40). 
Journal of Function Spaces and Applications

5

One can easily check that if we put $n=2$ in Theorem 6 then we get the following.

Corollary 7 (see [5]). Let $p, q>1$ and let $f, g$ be non-negative functions on $[a, b]$ such that $f^{p}, g^{q}$ are concave on $[a, b]$. Then

$$
\frac{(f(a)+f(b))^{p}(g(a)+g(b))^{q}}{2^{p+q}} \leq \frac{1}{(b-a)^{2}}\|f\|_{p}^{p}\|g\|_{q}^{q} .
$$

Theorem 8. Let $n$ be a positive integer such that $n \geq 2$ and $p_{1}, p_{2}, \ldots, p_{n}>1, \prod_{i=1}^{n}\left(1 / p_{i}\right)=1$, and let $f_{1}, f_{2}, \ldots, f_{n}$ be positive functions on $[a, b]$ such that the function $f_{i}^{p_{i}}$ is integrable on $[a, b], 0<\int_{a}^{b} f_{i}^{p_{i}}(x) d x<\infty$ for all $i=1,2, \ldots$, $n$, and

$$
0<m_{i} \leq \frac{f_{i}(x)}{f_{i+1}(x)} \leq M_{i}
$$

for all $x \in[a, b]$ and $i=1, \ldots, n-1$. Then

$$
\int_{a}^{b}\left(\prod_{i=1}^{n} f_{i}(x)\right) d x \leq \sum_{i=1}^{n}\left(\frac{s_{i}}{p_{i}}\left(\sum_{j=1}^{n}\left\|f_{j}\right\|_{p_{i}}^{p_{i}}\right)\right)
$$

where

$$
\begin{gathered}
s_{1}=2^{p_{1}-1}\left(\frac{M_{1}}{M_{1}+1}\right)^{p_{1}}, \quad s_{n}=2^{p_{n}-1}\left(\frac{1}{m_{n-1}+1}\right)^{p_{n}}, \\
s_{i}=\min \left\{2^{p_{i}-1}\left(\frac{1}{m_{i-1}+1}\right)^{p_{i}}, 2^{p_{i}-1}\left(\frac{M_{i}}{M_{i}+1}\right)^{p_{i}}\right\}
\end{gathered}
$$

for all $1<i<n$.

Proof. By Lemma 1, we have

$$
\begin{aligned}
& \left(\int_{a}^{b} f_{i}^{p_{i}}(x) d x\right)^{1 / p_{i}} \\
& \quad \leq \frac{M_{i}}{M_{i}+1}\left(\int_{a}^{b}\left(f_{i}(x)+f_{i+1}(x)\right)^{p_{i}} d x\right)^{1 / p_{i}}, \\
& \left(\int_{a}^{b} f_{i+1}^{p_{i+1}}(x) d x\right)^{1 / p_{i+1}} \\
& \quad \leq \frac{1}{m_{i}+1}\left(\int_{a}^{b}\left(f_{i}(x)+f_{i+1}(x)\right)^{p_{i+1}} d x\right)^{1 / p_{i+1}}
\end{aligned}
$$

for all $i=1, \ldots, n-1$.
Using the elementary inequality $(\alpha+\beta)^{p} \leq 2^{p-1}\left(\alpha^{p}+\beta^{p}\right)$ where $p>1$ and $\alpha, \beta>0$, we get

$$
\begin{aligned}
\int_{a}^{b} f_{i}^{p_{i}}(x) d x & \leq\left(\frac{M_{i}}{M_{i}+1}\right)^{p_{i}} \int_{a}^{b}\left(f_{i}(x)+f_{i+1}(x)\right)^{p_{i}} d x \\
& \leq\left(\frac{M_{i}}{M_{i}+1}\right)^{p_{i}} \int_{a}^{b} 2^{p_{i}-1}\left(f_{i}^{p_{i}}(x)+f_{i+1}^{p_{i}}(x)\right) d x, \\
\int_{a}^{b} f_{i+1}^{p_{i+1}}(x) d x & \leq\left(\frac{1}{m_{i}+1}\right)^{p_{i+1}} \int_{a}^{b}\left(f_{i}(x)+f_{i+1}(x)\right)^{p_{i+1}} d x \\
& \leq\left(\frac{1}{m_{i}+1}\right)^{p_{i+1}} \int_{a}^{b} 2^{p_{i+1}-1} \\
\times & \times\left(f_{i}^{p_{i+1}}(x)+f_{i+1}^{p_{i+1}}(x)\right) d x
\end{aligned}
$$

for all $i=1, \ldots, n-1$.

Then

$$
\begin{aligned}
\int_{a}^{b} f_{i}^{p_{i}}(x) d x & \leq 2^{p_{i}-1}\left(\frac{M_{i}}{M_{i}+1}\right)^{p_{i}} \int_{a}^{b}\left(\sum_{j=1}^{n} f_{j}^{p_{i}}(x)\right) d x \\
& =2^{p_{i}-1}\left(\frac{M_{i}}{M_{i}+1}\right)^{p_{i}} \sum_{j=1}^{n}\left(\int_{a}^{b} f_{j}^{p_{i}}(x) d x\right), \\
\int_{a}^{b} f_{i+1}^{p_{i+1}}(x) d x & \leq 2^{p_{i+1}-1}\left(\frac{1}{m_{i}+1}\right)^{p_{i+1}} \int_{a}^{b}\left(\sum_{j=1}^{n} f_{j}^{p_{i+1}}(x)\right) d x \\
& =2^{p_{i+1}-1}\left(\frac{1}{m_{i}+1}\right)^{p_{i+1}} \sum_{j=1}^{n}\left(\int_{a}^{b} f_{j}^{p_{i+1}}(x) d x\right)
\end{aligned}
$$

for all $i=1, \ldots, n-1$.

Let $s_{1}=2^{p_{1}-1}\left(M_{1} /\left(M_{1}+1\right)\right)^{p_{1}}, s_{n}=2^{p_{n}-1}\left(1 /\left(m_{n-1}+1\right)\right)^{p_{n}}$, and

$$
s_{i}=\min \left\{2^{p_{i}-1}\left(\frac{1}{m_{i-1}+1}\right)^{p_{i}}, 2^{p_{i}-1}\left(\frac{M_{i}}{M_{i}+1}\right)^{p_{i}}\right\}
$$

for all $1<i<n$.

It follows that

$$
\begin{aligned}
& \int_{a}^{b} f_{1}^{p_{1}}(x) d x \leq s_{1} \sum_{j=1}^{n}\left(\int_{a}^{b} f_{j}^{p_{1}}(x) d x\right), \\
& \int_{a}^{b} f_{n}^{p_{n}}(x) d x \leq s_{n} \sum_{j=1}^{n}\left(\int_{a}^{b} f_{j}^{p_{n}}(x) d x\right), \\
& \int_{a}^{b} f_{i}^{p_{i}}(x) d x \leq s_{i} \sum_{j=1}^{n}\left(\int_{a}^{b} f_{j}^{p_{i}}(x) d x\right)
\end{aligned}
$$

for all $1<i<n$. 
By the generalized Young inequality, we obtain that

$$
\begin{aligned}
\int_{a}^{b}\left(\prod_{i=1}^{n} f_{i}(x)\right) d x & \leq \int_{a}^{b}\left(\sum_{i=1}^{n} \frac{1}{p_{i}} f_{i}^{p_{i}}(x)\right) d x \\
& =\sum_{i=1}^{n} \frac{1}{p_{i}}\left(\int_{a}^{b} f_{i}^{p_{i}}(x) d x\right) \\
& \leq \sum_{i=1}^{n} \frac{1}{p_{i}}\left(s_{i} \sum_{j=1}^{n}\left(\int_{a}^{b} f_{j}^{p_{i}}(x) d x\right)\right) \\
& \leq \sum_{i=1}^{n}\left(\frac{s_{i}}{p_{i}}\left(\sum_{j=1}^{n}\left\|f_{j}\right\|_{p_{i}}^{p_{i}}\right)\right)
\end{aligned}
$$

This proof is completed.

Applying Theorem 8 with $n=2$ and putting there $p_{1}=$ $p, p_{2}=q, 2 s_{1} / p_{1}=c_{1}$, and $2 s_{2} / p_{2}=c_{2}$, we get the following.

Corollary 9 (see [5]). Let $p, q>1,1 / p+1 / q=1$, and let $f$ and $g$ be positive functions on $[a, b]$ such that $0<\int_{a}^{b}$ $f^{p}(x) d x<\infty, 0<\int_{a}^{b} g^{q}(x) d x<\infty$, and

$$
0<m \leq \frac{f(x)}{g(x)} \leq M
$$

for all $x \in[a, b]$. Then

$$
\int_{a}^{b} f(x) g(x) d x \leq c_{1}\left(\frac{\|f\|_{p}^{p}+\|g\|_{p}^{p}}{2}\right)+c_{2}\left(\frac{\|f\|_{q}^{q}+\|g\|_{q}^{q}}{2}\right),
$$

where

$$
c_{1}=\frac{2^{p}}{p}\left(\frac{M}{M+1}\right)^{p}, \quad c_{2}=\frac{2^{q}}{q}\left(\frac{1}{m+1}\right)^{q} .
$$

\section{Acknowledgment}

The author would like to thank the referees for their useful comments and suggestions.

\section{References}

[1] B. G. Pachpatte, "On some inequalities for convex functions," RGMIA Research Report Collection, vol. 6, 9 pages, 2003.

[2] B. G. Pachpatte, "A note on integral inequalities involving two log-convex functions," Mathematical Inequalities \& Applications, vol. 7, no. 4, pp. 511-515, 2004.

[3] U. S. Kirmaci, M. Klaričić Bakula, M. E. Özdemir, and J. Pečarić, "Hadamard-type inequalities for $s$-convex functions," Applied Mathematics and Computation, vol. 193, no. 1, pp. 26-35, 2007.

[4] M. K. Bakula, M. E. Özdemir, and J. Pečarić, "Hadamard type inequalities for $m$-convex and $(\alpha, m)$-convex functions," Journal of Inequalities in Pure and Applied Mathematics, vol. 9, no. 4, article 96, 12 pages, 2008.
[5] E. Set, M. E. Özdemir, and S. S. Dragomir, "On the HermiteHadamard inequality and other integral inequalities involving two functions," Journal of Inequalities and Applications, vol. 2010, Article ID 148102, 9 pages, 2010.

[6] D. S. Mitrinović, J. E. Pečarić, and A. M. Fink, Classical and New Inequalities in Analysis, vol. 61 of Mathematics and Its Applications, Kluwer Academic Publishers, Dodrecht, The Netherlands, 1993.

[7] M. Alomari and M. Darus, "On the Hadamard's inequality for log-convex functions on the coordinates," Journal of Inequalities and Applications, vol. 2009, Article ID 283147, 13 pages, 2009.

[8] C. Dinu, "Hermite-Hadamard inequality on time scales," Journal of Inequalities and Applications, vol. 2008, Article ID 287947, 24 pages, 2008.

[9] J. Pečarić and T. Pejković, “On an integral inequality," Journal of Inequalities in Pure and Applied Mathematics, vol. 5, no. 2, article 47, 6 pages, 2004.

[10] J. E. Pečarić, F. Proschan, and Y. L. Tong, Convex Functions, Partial Orderings, and Statistical Applications, vol. 187 of Mathematics in Science and Engineering, 1992.

[11] T. K. Pogány, "On an open problem of F. Qi," Journal of Inequalities in Pure and Applied Mathematics, vol. 3, no. 4, article 54, 5 pages, 2002.

[12] P. S. Bullen, D. S. Mitrinović, and P. M. Vasić, Means and Their Inequalities, vol. 31 of Mathematics and Its Applications, D. Reidel Publishing, Dordrecht, The Netherlands, 1988.

[13] L. Bougoffa, "On Minkowski and Hardy integral inequalities," Journal of Inequalities in Pure and Applied Mathematics, vol. 7, no. 2, article 60, 3 pages, 2006. 


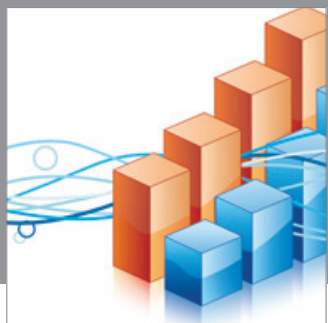

Advances in

Operations Research

mansans

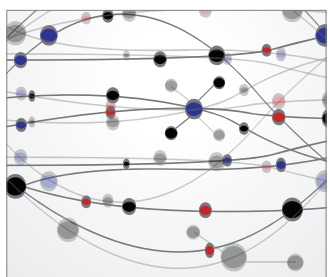

The Scientific World Journal
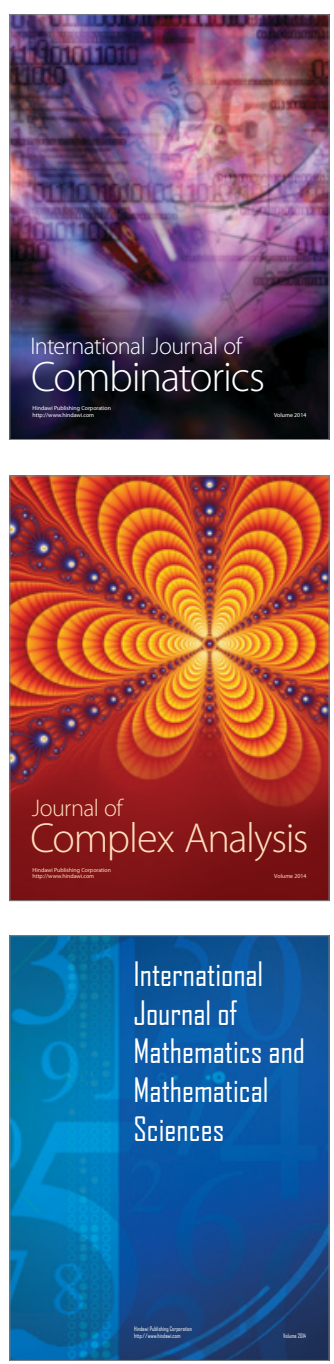
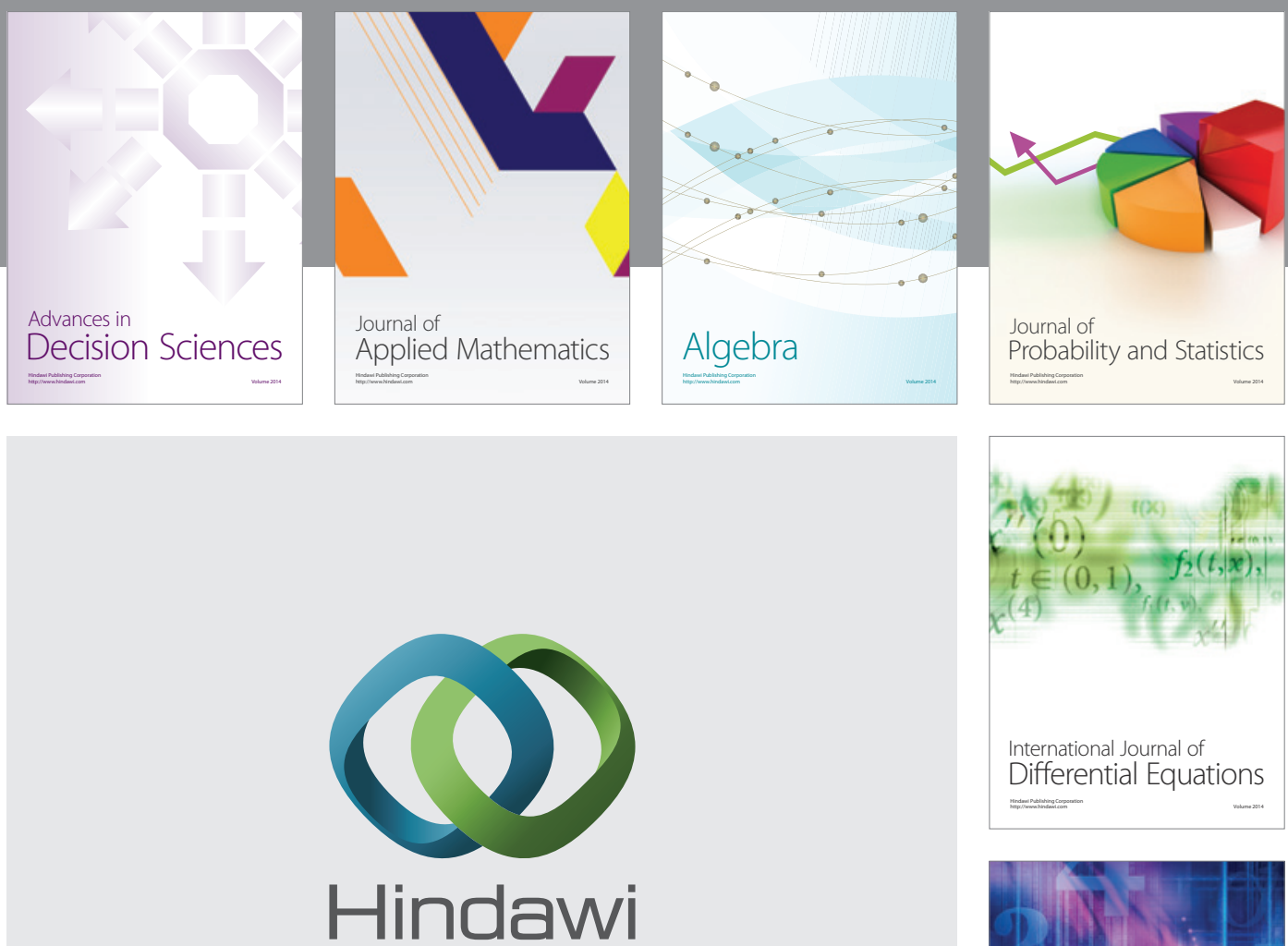

Submit your manuscripts at http://www.hindawi.com
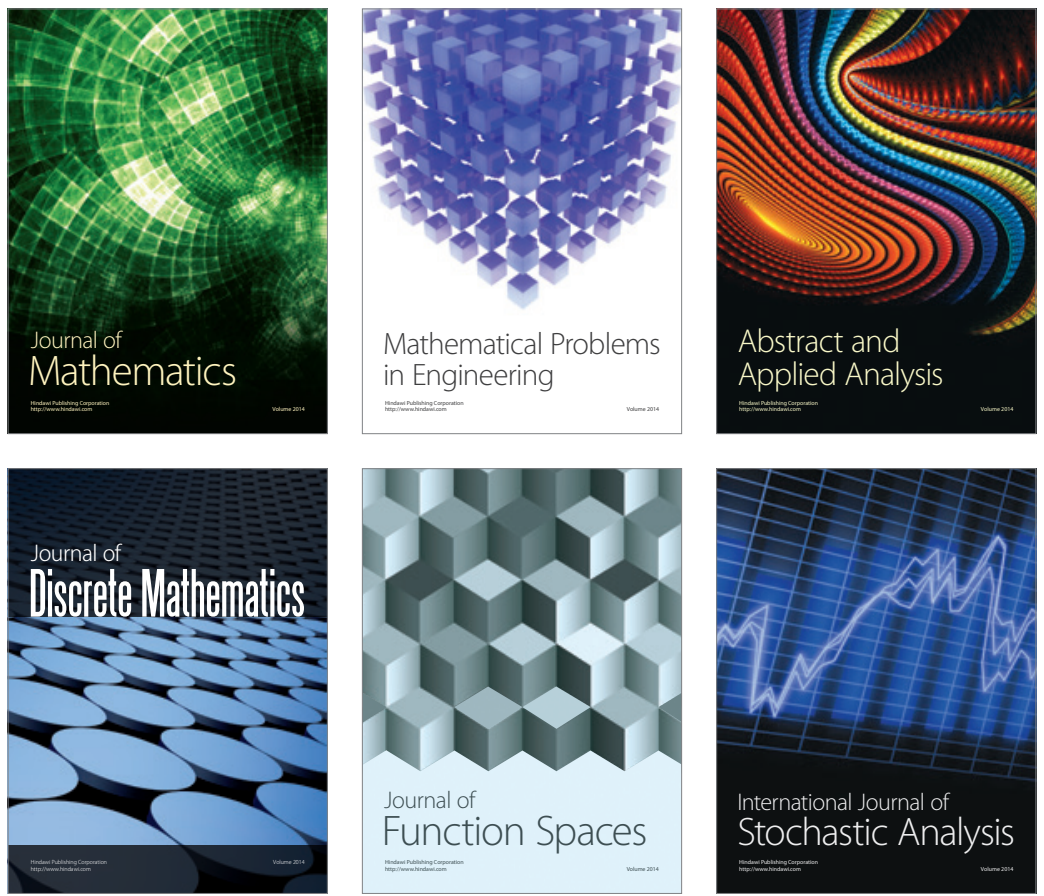

Journal of

Function Spaces

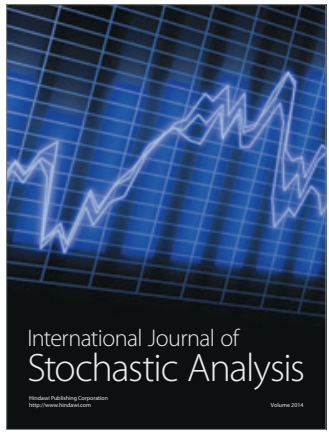

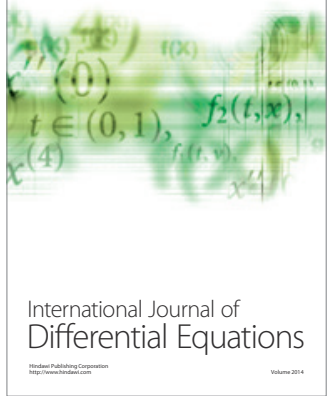
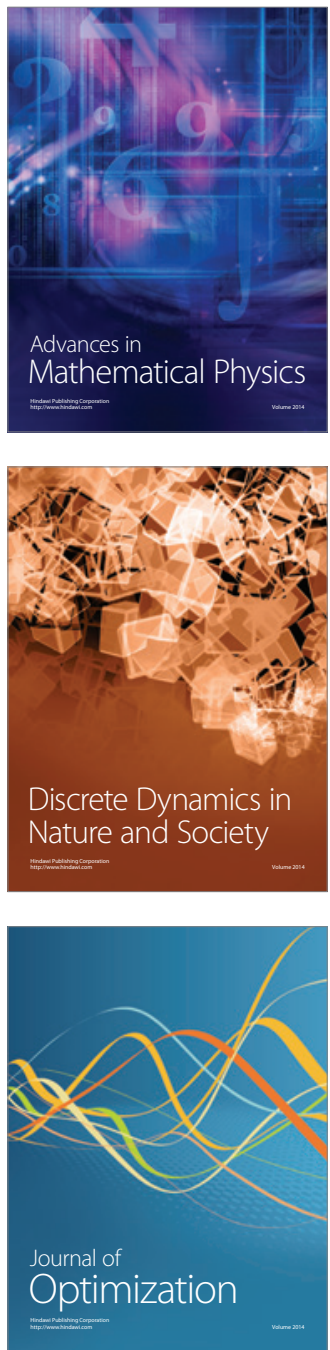